\title{
Transplant outcomes in positive complement-dependent cytotoxicity- versus flow cytometry-crossmatch kidney transplant recipients after successful desensitization: a retrospective study
}

Deok Gie Kim', Juhan Lee ${ }^{2}$, Younhee Park ${ }^{3}$, Myoung Soo Kim², ${ }^{2,}$ Hyeon Joo Jeong ${ }^{4,5}$, Soon II Kim²,4, Yu Seun Kim ${ }^{2,4}$, Beom Seok Kim ${ }^{4,6^{*}}$ and Kyu Ha Huhn $2,4^{*}$

\begin{abstract}
Background: Despite the obvious survival benefit compared to that among waitlist patients, outcomes of positive crossmatch kidney transplantation (KT) are generally inferior to those of human leukocyte antigen (HLA)-compatible $K T$. This study aimed to compare the outcomes of positive complement-dependent cytotoxicity (CDC) crossmatch $(\mathrm{CDC}+\mathrm{FC}+)$ and positive flow cytometric crossmatch (CDC-FC+) with those of HLA-compatible KT (CDC-FC-) after successful desensitization.

Methods: We retrospectively analyzed 330 eligible patients who underwent KTs between June 2011 and August 2017: CDC-FC- $(n=274), C D C-F C+(n=39)$, and CDC + FC+ $(n=17)$. Desensitization protocol targeting donorspecific antibody (DSA) involved plasmapheresis, intravenous immunoglobulin (IVIG), and rituximab with/without bortezomib for positive-crossmatch KT.

Results: Death-censored graft survival and patient survival were not different among the three groups. The median estimated glomerular filtration rate was significantly lower in the $C D C+F C+$ group than in the compatible group at 6 months $(P<0.001)$ and 2 years $(P=0.020)$. Biopsy-proven rejection within 1 year of $C D C-F C-, C D C-F C+$, and $C D C+$ FC+ were 15.3, 28.2, and 47.0\%, respectively. Urinary tract infections $(P<0.001)$, Pneumocystis jirovecii pneumonia $(P<0.001)$, and cytomegalovirus viremia $(P<0.001)$ were more frequent in $C D C-F C+$ and $C D C+F C+$ than in CDCFC-

Conclusions: This study showed that similar graft and patient survival was achieved in CDC-FC+ and CDC + FC+ KT compared with CDC-FC- through DSA-targeted desensitization despite the higher incidence of rejection and infection than that in compatible KT.
\end{abstract}

Keywords: Kidney transplantation, Positive crossmatch, Donor-specific antibody, Desensitization

\footnotetext{
* Correspondence: DOCBSK@yuhs.ac; khhuh@yuhs.ac

${ }^{4}$ The Research Institute for Transplantation, Yonsei University College of

Medicine, Seoul, Republic of Korea

${ }^{2}$ Department of Surgery, Yonsei University College of Medicine, Seoul,

Republic of Korea

Full list of author information is available at the end of the article
}

(c) The Author(s). 2019 Open Access This article is distributed under the terms of the Creative Commons Attribution 4.0 International License (http://creativecommons.org/licenses/by/4.0/), which permits unrestricted use, distribution, and reproduction in any medium, provided you give appropriate credit to the original author(s) and the source, provide a link to the Creative Commons license, and indicate if changes were made. The Creative Commons Public Domain Dedication waiver (http://creativecommons.org/publicdomain/zero/1.0/) applies to the data made available in this article, unless otherwise stated. 


\section{Background}

Patients undergoing positive-crossmatch kidney transplantation (KT) from living donors have the advantage of survival compared to highly sensitized patients on waitlists [1]. However, there is inevitable immunologic risk despite rigorous pre- or posttransplant immunosuppression during desensitization. Several studies have reported a higher graft failure and mortality after positive-crossmatch KT than after compatible KT [2-4]; hence, only certain centers perform positive-crossmatch KT in the United States [5].

Methods based on complement-dependent cytotoxicity (CDC) crossmatch for identifying and characterizing anti-HLA antibody, such as the single-antigen assay and the $\mathrm{C} 1 \mathrm{q}$ assay, have recently been developed and used clinically $[6,7]$. Since the introduction of more sensitive methods such as flow cytometry (FC) crossmatch and the single antigen assay (SAA) using Luminex technology, the importance of quantifying antigen strength has been emphasized [6, 8-12]. Furthermore, baseline donor-specific antibody (DSA) titers are associated with the outcome of positive-crossmatch KT [13]. However, most studies on positive-crossmatch KT so far did not examine preoperative DSA titers, but rather involved retrospective examinations, thus not indicating DSA to be an appropriate target for successful desensitization.

A recent study reported a similar graft and patient survival rate between positive-crossmatch living-donor $\mathrm{KT}$ (LDKT) and compatible KT upon desensitization with a risk-stratified protocol [14]; however, the study involved a small cohort undergoing CDC-positive KT. Hence, this study aimed to evaluate post-transplant outcomes of CDC-positive $(\mathrm{CDC}+\mathrm{FC}+)$ and FC-positive (CDC-FC+) $\mathrm{KT}$ using a DSA-targeted desensitization protocol and compare these outcomes with those of compatible KT (CDC-FC-).

\section{Methods}

\section{Study population}

We retrospectively assessed 691 consecutive living donor KT recipients at Severance hospital in Seoul, Korea from June 2011, when Luminex-based SAA was initiated, to August 2017. Patients under 18 years of age, those receiving kidney transplants from HLA-identical siblings, $\mathrm{ABO}$ incompatible $\mathrm{KT}$, those taking cyclosporin primarily as an immunosuppressant, and those lacking findings of pretransplant DSA or FC were excluded. Cases of negative crossmatch with DSA $(n=21)$ were excluded in accordance with the recent report on non-inferior graft survival [15]. Furthermore, patients presenting positive $\mathrm{T}$-cell but negative B-cell crossmatches and positive FC without DSA were excluded owing to potential technical errors. Finally, 330 eligible patients were divided into three groups; CDC-FC-, $\mathrm{CDC}-\mathrm{FC}+$, and $\mathrm{CDC}+\mathrm{FC}+$ (Fig. 1).

\section{Crossmatch and detection of DSA}

Throughout the study, CDC and FC crossmatch and SAA were screened for all KT recipients with their potential living donors and repeated 1 or 2 days before transplantation. T-cell CDC was performed with antihuman globulin test and B-cell CDC was performed by warm-method. The results were recorded as the maximum titer presenting more than $11 \%$ of cell lysis. FC crossmatch using FACS Canto II (Beckton Dickinson, San Jose, CA, USA) was considered positive when the median fluorescence intensity (MFI) ratio was greater than 2.0. SAA was performed using two reagents during the study period: 1. Lifecodes LSA class I and class II (Gen-Probe Transplant Diagnostics, Inc., Stamford, CT, USA) from Jun 2011 to Dec 2014; 2. LABScreen Single Antigen class I and class II (One Lambda, Canoga Park,

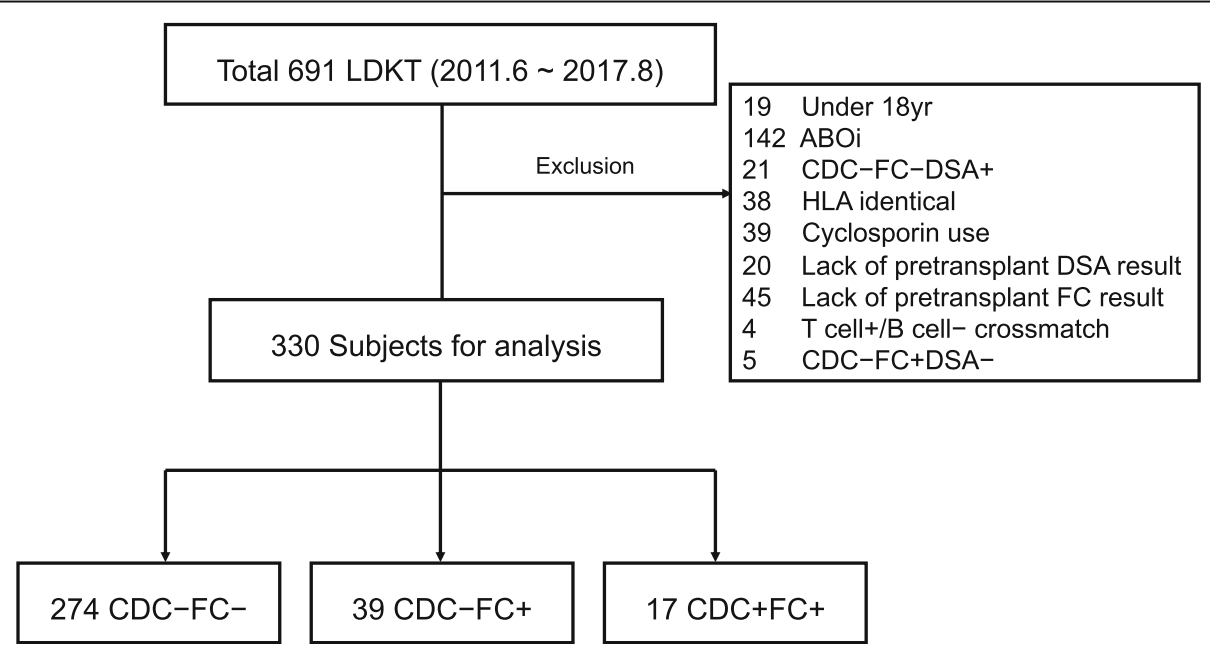

Fig. 1 Study population. CDC, complement dependent cytotoxicity; DSA, donor specific antibody; FC, flow cytometry; HLA, human leukocyte antigen; LDKT, living donor kidney transplantation 
CA, USA) from Jan 2015 to Aug 2017. Antibodies against donor HLA-A, B, DR, and DQ were characterized, and the strength of each DSA was determined at the maximum MFI value. An MFI of > 1000 was considered positive. We assessed DSA in all recipients before KT. After transplantation, DSA was checked only when rejection was suspected until September 2015 due to cost issue. Since then, SAA for the detection of DSA has been covered by national insurance in our country and we checked DSA at least once a year regardless of acute rejection. For CDC-FC+ and CDC + FC+ groups, routine DSA assessment was performed more frequently according to pretransplant antigen strength and clinical course, at least every 3 months during 1 posttransplant year. C1q binding ability of anti-HLA DSA was examined in accordance with a previously reported method (One Lambda) [7].

\section{Desensitization protocols and immunosuppression}

Pretransplant desensitization for positive-crossmatch KT at our institution involves plasmapheresis (PP)/low dose intravenous immunoglobulin (IVIG, $100 \mathrm{mg} / \mathrm{kg}$ per cycle) and rituximab $\left(375 \mathrm{mg} / \mathrm{m}^{2}\right)$ (Additional file 1: Figure S1 and Additional file 2: Figure S2). For CDC-FC+, PP/IVIG were administered 3-4 times 1 week before surgery. Negative conversion upon FC was considered an indicator of successful desensitization. However, rituximab usually results in false-positive B-cell crossmatch. Therefore, decisions regarding successful desensitization were primarily based on the outcomes of the SAA, especially for patients with class II DSA.

For $\mathrm{CDC}+\mathrm{FC}+$, a 2-3-week desensitization schedule was designed on the basis of the initial CDC titer and DSA titer. Rituximab $\left(375 \mathrm{mg} / \mathrm{m}^{2}\right)$ and PP/IVIG were administered 3 or 4 times a week, and the immunologic status was re-assessed via repeated CDC and SAA, with appropriate modifications in the desensitization schedule. The targets were T-cell CDC-negativity and an MFI $<5000$ of immunodominant DSA during the initial period. However, we subsequently progressed to transplantation with a much higher MFI value of DSA if enough responsiveness to desensitization was confirmed through negative conversion of crossmatch or C1q binding activity. Since June 2015, bortezomib, a proteasome inhibitor, has been administered to $\mathrm{CDC}+\mathrm{FC}+$ patients who did not respond to rituximab and 3-4 doses of PP/ IVIG. Four doses of bortezomib were administered (1.3 $\mathrm{mg} / \mathrm{m}^{2}$ per dose) for 2 weeks during desensitization. Usually, total $6-10$ cycles of PP with IVIG $(100 \mathrm{mg} / \mathrm{kg}$ per cycle) were performed for $\mathrm{CDC}+\mathrm{FC}+$ patients before KT.

Instead of basiliximab which is used for induction in negative-crossmatch KT, we routinely used antithymocyte globulin (ATG; $1.5 \mathrm{mg} / \mathrm{kg}$ ) for $1-4$ days after
KT for positive-crossmatch KTs. Alike negativecrossmatch $\mathrm{KT}$, tacrolimus-based triple immunosuppressive drugs were used for maintenance (tacrolimus, mycophenolate mofetil [MMF] and steroid), and the target trough levels of tacrolimus were $5-10 \mathrm{ng} / \mathrm{ml}$ during the first month after KT, followed by 3-7 ng/ml. MMF was initiated before transplantation at an initial dose of $1000-2000 \mathrm{mg} / \mathrm{d}$. Steroid was administered from the day of transplantation at $500-1000 \mathrm{mg}$ of intravenous methylprednisolone; thereafter, the dosage was tapered to that of oral prednisolone.

Using these protocols, we performed desensitization in $21 \mathrm{CDC}+\mathrm{FC}+$ patients, but 4 were not able to undergo $\mathrm{KT}$ because of a sustained high titer of T-cell CDC or $\mathrm{C} 1 \mathrm{q}$ positivity despite bortezomib administration. None of the CDC-FC+ patients failed to undergo KT.

\section{Analysis of clinical outcomes}

The primary end points were death-censored graft survival and patient survival. Graft failure was defined as returning to dialysis or renal re-transplantation. Secondary end points were biopsy-proven rejection (BPAR), graft function and infectious complications. Renal biopsy was performed only when graft rejection was clinically suspected based on increased serum creatinine or proteinuria, in accordance with our hospital policy. BPAR was confirmed by transplant pathologists at our institution and antibody mediated rejection (ABMR) or T-cell mediated rejection (TCMR) was diagnosed in accordance with Banff 2007 classification [16]. Allograft function was assessed on the basis of eGFR calculated using the Chronic Kidney Disease Epidemiology formula [17]. For comparisons at each time point, we considered eGFRs of the patients with graft failure as zero regardless of their follow-up status to minimize bias.

We routinely screened for cytomegalovirus (CMV) viremia (CMV DNA > 1000 copies/ml) and $\mathrm{BK}$ virus $(\mathrm{BKV})$ viremia $(\mathrm{BKV}$ DNA > 10,000 copies/ml) every 36 months for the first year and then annually among all patients. CMV was checked whenever the patient had fever. In case of positive-crossmatch KT, CMV was monitored every week for 1 month, then every 3-6 months unless CMV viremia was confirmed. No routine prophylaxis against CMV infections was administered but CMV was managed by preemptive strategy [18]. Prophylactic trimethoprim-sulfamethoxazole was administered for Pneumocystis jirovecii pneumonia (PJP) for a minimum of 6 months. For fungal prophylaxis, $4 \mathrm{ml}$ nystatin was orally administered four times a day for 12 months. Urinary tract infection (UTI), pneumonia, bacteremia, and intraabdominal infection were considered only when pathogens were identified with correlated symptoms. Herpes zoster was diagnosed on the basis of typical skin lesions. 


\section{Statistical analysis}

Data of categorical variables were shown as numbers (frequencies), and chi square test or Fisher's exact test were used when appropriate. For the comparison of continuous variables, the Kruskal-Wallis test was used, and data were expressed as median (interquartile range $[\mathrm{IQR}])$. Post hoc analysis was conducted with Bonferroni's method for the inter-group comparison of eGFR. To confirm the independent association between crossmatch positivity and eGFR, linear regression was applied for confounders. Survival outcomes were compared by Kaplan-Meier survival curves with log-rank tests and also adjusted using cox regression analysis. All analyses were performed using a standard software (SPSS v23.0; IBM, Armonk, NY, USA), and $P<0.05$ was considered statistically significant.

\section{Results}

\section{Baseline characteristics of patients}

Among 330 LDKT patients, 274 were CDC-FC-, 39 were $\mathrm{CDC}-\mathrm{FC}+$, and 17 were $\mathrm{CDC}+\mathrm{FC}+$. As shown in Table 1, age of recipients and donors was similar in all groups. Recipients in the $\mathrm{CDC}-\mathrm{FC}+$ and $\mathrm{CDC}+\mathrm{FC}+$ groups displayed female predominance compared to those in the control group and vice versa with respect to donor sex. The duration of pretransplant dialysis was similar; however, it was greater in cases of retransplantation in $\mathrm{CDC}-\mathrm{FC}+$ and $\mathrm{CDC}+\mathrm{FC}+$ groups than in the control group. Pretransplant diabetes and cardiovascular disease, which defined as myocardial infarction, coronary artery revascularization and stroke, showed similar incidence among the three groups.

\section{Immunologic status}

Table 2 shows the immunologic status before and after desensitization in positive-crossmatch groups. In the

Table 1 Baseline characteristics

\begin{tabular}{lllll}
\hline Variables & $\begin{array}{l}\mathrm{CDC}-\mathrm{FCM}- \\
(n=274)\end{array}$ & $\begin{array}{l}\mathrm{CDC}-\mathrm{FCM}+ \\
(n=39)\end{array}$ & $\begin{array}{l}\mathrm{CDC}+\mathrm{FC}+ \\
(n=17)\end{array}$ & $P$ \\
\hline Age, years & $48(36-55)$ & $52(45-57)$ & $46(41-56)$ & 0.059 \\
Sex, males & $178(65.0 \%)$ & $6(15.4 \%)$ & $4(2.1 \%)$ & $<0.001$ \\
Donor age, years & $45(34-52)$ & $43(33-53)$ & $41(29-49)$ & 0.647 \\
Donor sex, males & $111(40.5 \%)$ & $24(61.5 \%)$ & $5(29.4 \%)$ & 0.004 \\
Dialysis duration & & & & 0.228 \\
$\quad$ Preemptive & $95(34.7 \%)$ & $11(28.2 \%)$ & $4(23.5 \%)$ & \\
$\quad \leq 1$ year & $118(43.1 \%)$ & $13(33.3 \%)$ & $8(47.1 \%)$ & \\
$\quad>1$ year & $61(22.3 \%)$ & $15(38.5 \%)$ & $5(29.4 \%)$ & \\
Retransplantation & $12(4.4 \%)$ & $6(15.4 \%)$ & $5(29.4 \%)$ & $<0.001$ \\
Pretransplant DM & $73(26.0 \%)$ & $13(33.3 \%)$ & $2(11.8 \%)$ & 0.245 \\
Pretransplant CVD & $20(7.3 \%)$ & $3(7.7 \%)$ & $1(5.9 \%)$ & 0.971 \\
\hline
\end{tabular}

CDC Complement dependent cytotoxicity, FC Flow cytometry
CDC-FC+ group, $53.9 \%$ patients were positive for B-cell FC, and $46.1 \%$ patients were positive for both $\mathrm{T}$ - and Bcell FC. The median MFI ratio was 3.6 (maximum 18.3) for T-cell FC and 8.0 (maximum 53.3) for B-cell FC. Before desensitization, there was a median of 2 DSA specificities per patient (IQR, 1-3), and the most frequent type of immunodominant DSA was anti-HLA DR (56.4\%). Median MFI for immunodominant DSA was 4219 (IQR, 2357-10,000; maximum, 12,802). After desensitization with a median of three doses of PP/IVIG (IQR, 3-4), DSA was obliterated in $30.8 \%$ patients, and the median MFI for immune-dominant DSA declined to 1902 (IQR, 0-4294; maximum, 11,979).

In the $\mathrm{CDC}+\mathrm{FC}+$ group, $64.7 \%$ patients were positive for $\mathrm{B}$-cell $\mathrm{CDC}$, and $35.3 \%$ patients were positive for both $\mathrm{T}$ - and B-cell CDC. The maximum titer of CDC positivity was 1:32 for both $\mathrm{T}$ - and B-cell crossmatches. There was a median of 5 DSA specificities per patient (IQR 5-6), and the most frequent type of immunodominant DSA was also anti-HLA DR (52.9\%). The median MFI for immunodominant DSA was 10,951 (IQR, 573214,724; maximum, 18,056). After desensitization with a median of 6 doses and maximum of 11 doses of PP/ IVIG, DSA was obliterated in $11.8 \%$ of recipients and the median MFI for immunodominant DSA decreased to 4379 (IQR, 1492-10,457; maximum, 19,235). We assessed the C1q-binding ability in 16 subsequent cases before and after desensitization. Nine patients presented C1q-binding DSA before desensitization. Two patients with sustained $\mathrm{C} 1 \mathrm{q}$ positivity after 1 or 2 weeks of desensitization received 4 doses of bortezomib before transplantation. Eventually, 8 of $9(88.8 \%)$ patients presented negative conversion of $\mathrm{C} 1 \mathrm{q}$ binding ability, although DSA was still positive with considerable MFI value (minimum, 2259; maximum, 19,235).

\section{Graft and patient survival}

During a median follow-up of 37 (IQR, 22-52) months, 12 cases of death-censored graft failure were noted. The causes of graft failure were acute ABMR (5 CDC-FC-, 1 CDC-FC+), chronic ABMR (2 CDC-FC-, 1 CDC-FC+, and $1 \mathrm{CDC}+\mathrm{FC}+$ ), acute tubular injury (1 CDC-FC-), or non-compliance (1 CDC-FC-). Four patients died of acute cerebral infarction $(n=1)$, gastrointestinal bleeding $(n=2)$, or hematologic malignancy $(n=1)$.

On Kaplan Meier estimation of 5-year survival (Fig. 2), death-censored graft survival rates were $95.3 \%$ vs. $93.6 \%$ vs. $85.3 \%$ for CDC-FC-, CDC-FC+, and CDC + FC+, respectively, with no significant difference among them $(P=0.598)$. However, rejection-free graft survival was significantly lower in the $\mathrm{CDC}+\mathrm{FC}+$ group than in the other two groups $(80.1 \%$ vs. $73.5 \%$ vs. $34.7 \%$ for CDCFC-, CDC-FC+, and CDC + FC+, respectively; $P<0.001$ ). Patient survival did not differ significantly among the 
Table 2 Immunologic details before and after desensitization

\begin{tabular}{|c|c|c|}
\hline Variables & $\begin{array}{l}C D C-F C+ \\
(n=39)\end{array}$ & $\begin{array}{l}C D C+F C+ \\
(n=17)\end{array}$ \\
\hline FC positivity, \% (B / T and B) & $53.9 / 46.1$ & - \\
\hline \multicolumn{3}{|l|}{ FC MFI ratio } \\
\hline T cell positive $(n=21)$, median (IQR) & $3.6(2.9-6.8)$ Max 18.3 & - \\
\hline B cell positive $(n=39)$, median (IQR) & $8.0(4.4-17.4)$ Max 53.3 & - \\
\hline CDC positivity, \% (B / T and B) & - & $64.7 / 35.3$ \\
\hline \multicolumn{3}{|l|}{ CDC titer } \\
\hline T cell (AHG phase), number (1:2 / 1:4 / 1:8 / 1:32) & - & $2 / 1 / 2 / 1$ \\
\hline B cell (Warm phase), number (1:1 / 1:2 / 1:4 / 1:32) & - & $3 / 6 / 7 / 1$ \\
\hline Number of Pretransplant PP + IVIG, median (IQR) & 3 (3-4) Max 8 & $6(4-7)$ Max 11 \\
\hline \multicolumn{3}{|l|}{ Pre-desensitization DSA } \\
\hline HLA Class, \% (I / II /I + II) & $17.9 / 53.8 / 28.3$ & $17.6 / 35.3 / 47.1$ \\
\hline Number, median (IQR) & $2(1-3)$ & $5(5-6)$ \\
\hline Immunodominant DSA, \% (A / B / DR / DQ) & $12.8 / 15.4$ / $56.4 / 15.4$ & $11.9 / 17.6 / 52.9 / 17.6$ \\
\hline Immunodominant MFI, median (IQR) & 4219 (2357-10000) Max 12802 & 10951 (5732-14724) Max 18056 \\
\hline Sum of MFI, median (IQR) & 6577 (3686-13580) Max 45735 & 14663 (7818-24202) Max 66434 \\
\hline Positive for C1q binding assay & Not examined & 9 of 16, not examined in 1 \\
\hline \multicolumn{3}{|l|}{ Post-desensitization DSA } \\
\hline HLA Class, \% (None / I / II / I + II) & $30.8 / 12.8 / 46.2 / 10.3$ & $11.8 / 23.5 / 41.2 / 23.5$ \\
\hline Number, median (IQR) & $1(0-2)$ & $2(1-3)$ \\
\hline Immunodominant DSA, \% (None/A / B / DR / DQ) & $30.8 / 7.7 / 10.3 / 41.0 / 10.2$ & $11.8 / 11.8 / 11.8 / 52.8 / 11.8$ \\
\hline Immunodominant MFI, median (IQR) & 1902 (0-4294) Max 11979 & 4379 (1492-10457) Max 19235 \\
\hline Sum of MFI, median (IQR) & $2685(0-5811)$ Max 32811 & $5250(2264-15,844) \operatorname{Max} 36252$ \\
\hline Positive for C1q binding assay & Not examined & 0 of 16 , not examined in 1 \\
\hline
\end{tabular}

ATG Anti-thymocyte globulin, CDC Complement dependent cytotoxicity, DSA Donor-specific antibody, FC Flow cytometry, HLA Human leukocyte antigen, IVIG Intravenous immunoglobulin, MFI Median fluorescent intensity, PP Plasmapheresis

three groups $(P=0.524)$, although events were too small to draw statistical conclusion.

There was no significant risk factor for death-censored graft survival in Cox analysis so we adjusted it with covariates which showed difference in demographics among three groups such as age, sex, donor sex, retransplantation. Patient survival and rejection-free survival was adjusted with factors of which $P$ value was under 0.10 in univariate analysis; age and donor age for patient survival, donor age and dialysis duration for rejectionfree graft survival. After adjustment, crossmatch positivity was still not associated with death-censored graft survival and patient survival. However, $\mathrm{CDC}+\mathrm{FC}+$ versus CDC-FC- (HR 5.29, 95\% CI 2.84-987; $P<0.001$ ) was independent risk factor for rejection-free graft survival after adjustment. Full results of Cox analysis are provided by Additional file 3: Table S1-S3.

\section{Graft function}

Figure 3 shows the distribution of eGFR during 5 years of follow-up. The CDC-FC- and CDC-FC+ groups showed a median eGFR of $>60 \mathrm{ml} / \mathrm{min} / 1.73 \mathrm{~m}^{2}$ throughout the study period, although it slightly decreased with time. In the $\mathrm{CDC}+\mathrm{FC}+$ group, however, median eGFR declined to $<60$ at 3 years and $<40$ from the fourth year after KT. Furthermore, eGFRs of the $\mathrm{CDC}+\mathrm{FC}+$ group were lower than those of the other two groups throughout the follow-up period, although they differed significantly between the CDC-FC- and $\mathrm{CDC}+\mathrm{FC}+$ groups at 6 months $(P=0.037), 2$ years $(P=$ $0.038)$, and 3 years $(p=0.050)$. After adjustment with significantly associated factors in univariate analysis (sex, donor age and donor sex for eGFR at 6 months and 2 years; donor age for eGFR at 3 years), CDC + FC+ versus CDC-FC- was independently associated with eGFR at 6 months $(\beta=-8.18, P=<0.001)$ and that at 2 years $(\beta=$ $-5.85, P=0.020)$.

\section{Biopsy-proven acute rejection within 1 year after transplantation}

During posttransplant year 1, BPAR occurred in 42 CDC-FC- (15.3\%) patients, 11 CDC-FC+ (28.2\%) patients, and $8 \mathrm{CDC}+\mathrm{FC}+(47.0 \%)$ patients. Although the number of rejections of crossmatch positive groups was 


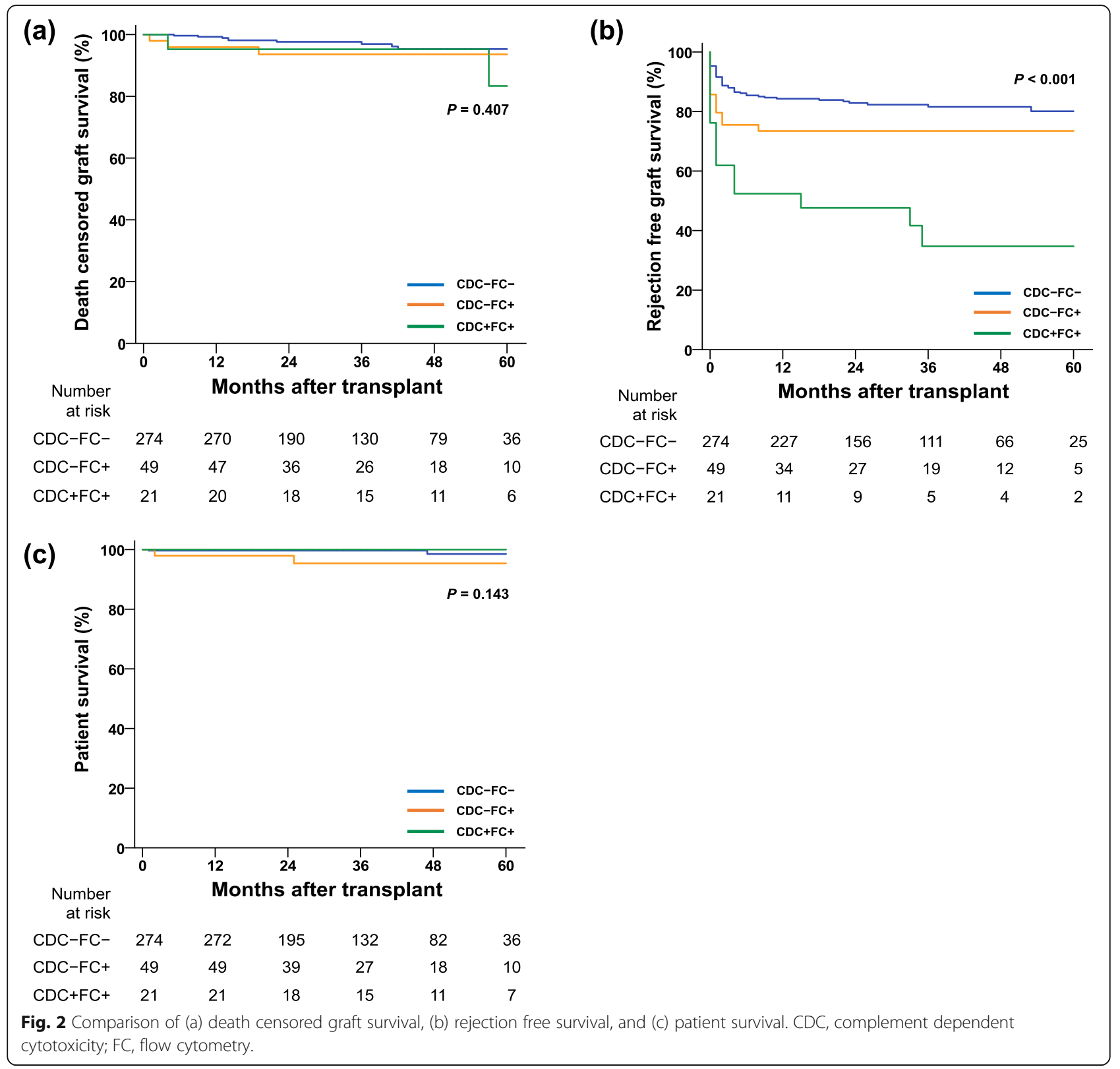

too small to compare statistically, details regarding the first occurrence of BPAR and corresponding rescue treatment are compared in Table 3. The median duration from KT to BPAR was significantly shorter among CDC-FC+ (9 days; IQR, 5-31) and CDC + FC+ (14 days; IQR, 5-38) patients than in CDC-FC- patients (42 days; IQR, 11-109; $P=0.035)$. Among BPARs, ABMR was 42.9\% and TCMR was $57.1 \%$ in CDC-FC- patients, while most types of rejections were ABMR in CDC-FC+ (90.9\%) and $\mathrm{CDC}+\mathrm{FC}+(100 \%)$. Over half of the cases of rejection in the CDC-FC- group received only a steroid pulse or additional ATG as rescue treatment, while most patients in the CDC-FC+ $(100 \%)$ and $\mathrm{CDC}+\mathrm{FC}+$ (75.0\%) groups received PP/IVIG. Three (27.3\%) patients in the CDC-FC+ and $5(62.5 \%)$ in the $\mathrm{CDC}+\mathrm{FC}+$ group received PP/IVIG along with 4 doses of bortezomib upon DSA detection at high MFI or severe deterioration of allograft function.

\section{Infectious complications within 1 year after} transplantation

As shown in Table 4, the occurrence rate of urinary tract infection $(7.7 \%$ vs. $51.3 \%$ vs. $23.5 \%, P<0.001)$ was significantly higher in CDC-FC+ and CDC + FC+ groups than in the controls. Bacteremia ( $2.6 \%$ vs. $12.8 \%$ vs. $0 \%)$ and intraabdominal infection $(0.7 \%$ vs. $7.7 \%$ vs. $0 \%, P=$ $0.036)$ were significantly more frequent in $\mathrm{CDC}-\mathrm{FC}+\mathrm{pa}-$ tients than in the other groups. $\mathrm{CDC}+\mathrm{FC}+$ patients 


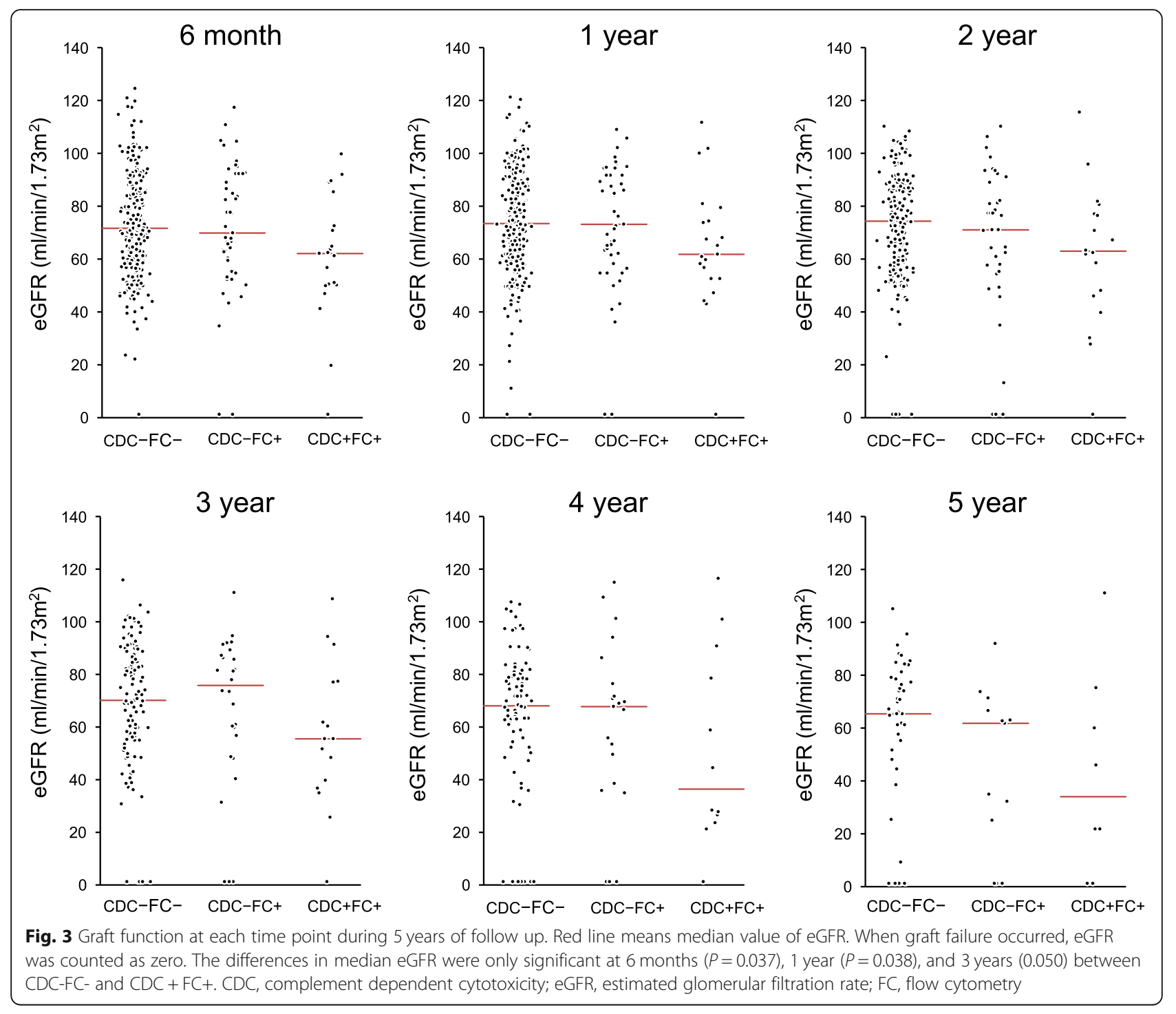

Table 3 Rejection pathology and rescue treatments in the patients who experienced biopsy proven rejection within 1 year after transplantation

\begin{tabular}{llll}
\hline Variables & $\begin{array}{l}\text { CDC }-\mathrm{FC}- \\
(n=42)\end{array}$ & $\begin{array}{l}\mathrm{CDC}-\mathrm{FC}+ \\
(n=11)\end{array}$ & $\begin{array}{l}\mathrm{CDC}+\mathrm{FC}+ \\
(n=8)\end{array}$ \\
\hline $\begin{array}{l}\text { Median time to BPAR (days) } \\
\text { Type of acute rejection }\end{array}$ & $42(11-109)$ & $9(5-31)$ & $14(5-38)$ \\
$\quad$ ABMR & $18(42.9 \%)$ & $10(90.9 \%)$ & $8(100 \%)$ \\
TCMR & $24(57.1 \%)$ & $1(9.1 \%)$ & $0(0 \%)$ \\
Rescue treatment & $16(38.1 \%)$ & & $2(25.0 \%)$ \\
$\quad$ Steroid pulse only & $7(16.7 \%)$ & 0 & 0 \\
Steroid pulse + ATG & $19(45.2 \%)$ & 0 & $1(12.5 \%)$ \\
PP/IVIG & 0 & $8(72.7 \%)$ & $5(62.5 \%)$ \\
PP/IVIG + bortezomib & $3(27.3 \%)$ & 0.001 \\
\hline
\end{tabular}


Table 4 Infectious complication within 1 year after transplantation

\begin{tabular}{lllll}
\hline Variables & $\begin{array}{l}\mathrm{CDC}-\mathrm{FC}- \\
(n=274)\end{array}$ & $\begin{array}{l}\mathrm{CDC}-\mathrm{FC}+ \\
(n=39)\end{array}$ & $\begin{array}{l}\mathrm{CDC}+\mathrm{FC}+ \\
(n=17)\end{array}$ & $P$ \\
\hline UTI & $21(7.7 \%)$ & $19(51.3 \%)$ & $4(23.5 \%)$ & $<0.001$ \\
Bacterial pneumonia & $4(1.5 \%)$ & $2(5.1 \%)$ & 0 & 0.234 \\
Bacteremia & $7(2.6 \%)$ & $5(12.8 \%)$ & 0 & 0.004 \\
Intraabdominal infection & $2(0.7 \%)$ & $3(7.7 \%)$ & 0 & 0.003 \\
BKV viremia & $18(6.6 \%)$ & $2(5.1 \%)$ & 0 & 0.527 \\
Herpes zoster & $20(7.3 \%)$ & $3(7.7 \%)$ & $5(29.4 \%)$ & 0.006 \\
PJP & 0 & $1(2.6 \%)$ & $2(11.8 \%)$ & $<0.001$ \\
CMV viremia & $38(13.9 \%)$ & $19(48.7 \%)$ & $11(64.7 \%)$ & $<0.001$ \\
\hline
\end{tabular}

$B K V$ BK virus, CDC Complement dependent cytotoxicity, CMV Cytomegalovirus, FC Flow cytometry, PCP Pneumocystis jirovecii pneumonia, UTI Urinary tract infection

experienced the highest rate of herpes zoster, PJP, and $\mathrm{CMV}$ infections, and $\mathrm{CDC}-\mathrm{FC}+$ patents also experienced more PJP and CMV infections than the controls (7.3\% vs. $7.7 \%$ vs. $29.4 \%, P=0.046$ for herpes zoster; $0 \%$ vs. $2.6 \%$ vs. $11.8 \%, P=0.001$ for PJP; $13.9 \%$ vs. $48.7 \%$ vs. $64.7 \%, P<0.001$ for $\mathrm{CMV}$ infection; all the rates above were for $\mathrm{CDC}-\mathrm{FC}-$ vs. $\mathrm{CDC}-\mathrm{FC}+$ vs. $\mathrm{CDC}+\mathrm{FC}+$, respectively). No patient died from posttransplant infection within 1 year.

\section{Discussion}

Although patients undergoing crossmatch-positive KT have the advantage of increased survival compared with waitlist patients on dialysis, most studies reported that the outcome was inferior to that of compatible KT [2-4, 19]. In particular, the $\mathrm{CDC}+\mathrm{FC}+$ group showed poor graft survival, which has led to only few institutions performing this procedure. Our institution has actively performed $\mathrm{CDC}+\mathrm{FC}+\mathrm{KT}$ and $\mathrm{CDC}-\mathrm{FC}+\mathrm{KT}$ and achieved graft survival comparable to that of compatible KT with more advanced DSA-targeted desensitization compared to that in the present study. However, higher graft rejection, followed by lower graft function, especially in $\mathrm{CDC}+\mathrm{FC}+$, still warrants attention.

Our desensitization protocol was based on comprehensive interpretation of solid phase assays such as SAA and $\mathrm{C} 1 \mathrm{q}$ assay as well as cell-based crossmatch. Marfo et al. [20] suggested a cutoff for transplantable MFI of DSA as $<5000$ in positive-crossmatch patients who underwent desensitization, which was also indicated at our center during the initial period. However, we successfully performed KT for patients with higher DSA levels after desensitization. Although rejection occurred more frequently, most events were controllable and only 3 positive-crossmatch patients $(4.2 \%)$ lost their grafts within 1 year owing to rejection. Furthermore, our indication for desensitization has been extended beyond the generally accepted criteria, being as high as T- or B-cell CDC-positivity titer of 1:32 and five median DSA types with maximum MFI of $>15,000$. Nevertheless, patient and graft survival of positive-crossmatch KT was almost parallel to that of compatible KT. This surprising improvement was achieved through our DSA-targeted desensitization protocol and rescue treatment for acute rejection, especially through a well-planned approach for patients at very high risk.

The C1q-binding antibody assay has been recently reported as an indicator of allograft survival and responsiveness to rejection treatment [7, 21-23]. From August 2013, we monitored the $\mathrm{C} 1 \mathrm{q}$ assay in all $\mathrm{CDC}+\mathrm{FC}+$ conversion trials and designed more extensive desensitization methods for C1q-positive patients. Furthermore, we utilized C1q-negative conversion as the target for successful desensitization and then progressed to KT.

Bortezomib has been introduced to effectively treat acute rejection in KT recipients [24-26]. Although it is uncertain whether bortezomib affects transplantability alone [27], bortezomib-based desensitization reportedly reduced anti-HLA DSA and increased the rate of KT in positive-crossmatch recipients [28]. During the study period, two patients with T- and B-cell CDC-positivity and C1q-binding DSA at high MFI level received 4 doses of bortezomib before transplantation. Despite the higher strength of DSA than that in patients who underwent $\mathrm{CDC}+\mathrm{FC}+\mathrm{KT}$ before, two patients receiving bortezomib not only displayed successful KT outcomes but also did not experience acute rejection within 1 year after KT. Recently, we have routinely administered bortezomib for $\mathrm{CDC}+\mathrm{FC}+\mathrm{KT}$, expecting increased transplantability and decreased posttransplant acute rejection.

Another strength of our study was the more detailed comparison of infectious complications than previous studies among different immunologic risk groups. Although aggressive immunosuppression is inevitable, limited information is available regarding infectious complications in positive-crossmatch KT. Two studies reported that viral infection was increased in positivecrossmatch KT patients; however, they did not contain a control group $[29,30]$. A recent multicenter study reported that the rates of readmission owing to infectious complications in both HLA-incompatible group and control subjects were virtually the same [31]. Furthermore, Okuda et al. [14] reported that bacterial and viral infections were not different between the positive- and negative-crossmatch KT groups. However, our study shows that various infections such as UTIs, bacteremia, PJP, and CMV infections were significantly more prevalent in the positive-crossmatch group than in the compatible group. Despite this increased risk of infection, $\mathrm{CDC}+\mathrm{FC}+$ and $\mathrm{CDC}-\mathrm{FC}+$ groups showed excellent 
patient survival. This suggests that positive-crossmatch KT potentially increases complications, however, they are controllable and do not increase patient mortality.

Unlike other studies, we excluded patients who showed CDC-FC- but had pretranspant DSA. The reasons were; 1$)$ relatively small number $(n=21), 2)$ recent meta-analysis demonstrating low level DSA detected by Luminex alone couldn't affect short to medium posttransplant outcome [15]. So, we thought that including those patients would make the analysis complicated rather than resulted in better risk stratification according to HLA antibody strength.

The limitation of our study is its retrospective singlecenter design with small population and small number of events. Also, we reported only a 5 year-outcome at maximum; hence, our results cannot be extrapolated to long-term setting. Finally, protocol biopsy was not performed in our institution, so we couldn't compare chronic pathological results such as chronic ABMR or chronic allograft nephropathy.

\section{Conclusions}

This study showed that intermediate graft and patient survival after $\mathrm{CDC}-\mathrm{FC}+$ and $\mathrm{CDC}+\mathrm{FC}+\mathrm{KT}$ were improved to levels abreast with those after compatible KT, using a recent DSA-targeted desensitization protocol. Higher rejection rates are still a demanding challenge; thus, improved and specialized desensitization with advanced immunosuppressive agents is needed.

\section{Supplementary information}

Supplementary information accompanies this paper at https://doi.org/10. 1186/s12882-019-1625-2.

Additional file 1: Figure S1. Desensitization protocol for CDC-FC+ KT

Additional file 2: Figure S2. Desensitization protocol for CDC + FC+ KT

Additional file 3: Table S1. Cox analysis for death-censored graft survival. Table S2. Cox analysis for patient survival. Table S3. Cox analysis for rejection-free graft survival.

\section{Abbreviations}

ABMR: Antibody-mediated graft rejection; ATG: Anti-thymocyte globulin; BKV: BK virus; BPAR: Biopsy-proven rejection; CDC: Complement dependent cytotoxicity; CMV: Cytomegalovirus; DSA: Donor-specific antibody; FC: Flow cytometry; HLA: Human leukocyte antigen; IVIG: Intravenous immunoglobulin; KT: Kidney transplantation; LDKT: Living donor kidney transplantation; MFI: Median fluorescent intensity; PJP: Pneumocystis jirovecii pneumonia; PP: Plasmapheresis; SAA: Single antigen assay; UTI: Urinary tract infection

\section{Acknowledgements}

The authors appreciate Haejin Kim, Sensor Sohn, Sangmi Choi, and Wooyoung Chung at the Department of Laboratory Medicine, Yonsei University College of Medicine for their support in transplant immunology laboratory investigations.

\section{Authors' contributions}

DGK, BSK, and KHH designed the study; DGK and JL acquired the data; DGK performed statistical analysis; KHH, MSK, HJJ, SIK, YP and YSK performed the experiments; $\mathrm{KHH}$ and BSK supervised the study. All authors have read and approved the manuscript.

Funding

No funding was obtained for this study.

\section{Availability of data and materials}

The datasets for this study are available from the corresponding author on the reasonable request.

\section{Ethics approval and consent to participate}

This study was conducted in accordance with the tenets of the Declaration of Helsinki and the Declaration of Istanbul and was approved by the Institutional Review Board of Severance Hospital, Yonsei University Health System (IRB No.: 4-2019-0092) with exemption for the informed consent due to the retrospective feature of this study. All kidneys were obtained by the department of transplantation surgery in Severance hospital and we did not use organs from prisoners.

\section{Consent for publication}

Not applicable.

\section{Competing interests}

The authors declare that they have no competing interests.

\section{Author details}

${ }^{1}$ Department of Surgery, Yonsei University Wonju College of Medicine, Wonju, Republic of Korea. ${ }^{2}$ Department of Surgery, Yonsei University College of Medicine, Seoul, Republic of Korea. ${ }^{3}$ Department of Laboratory Medicine, Yonsei University College of Medicine, Seoul, Republic of Korea. ${ }^{4}$ The Research Institute for Transplantation, Yonsei University College of Medicine, Seoul, Republic of Korea. ${ }^{5}$ Department of Pathology, Yonsei University College of Medicine, Seoul, Republic of Korea. ${ }^{6}$ Department of Internal Medicine, Yonsei University College of Medicine, Seoul, Republic of Korea.

Received: 29 April 2019 Accepted: 14 November 2019

Published online: 09 December 2019

\section{References}

1. Orandi BJ, Luo X, Massie AB, Garonzik-Wang JM, Lonze BE, Ahmed R, Van Arendonk KJ, Stegall MD, Jordan SC, Oberholzer J, et al. Survival benefit with kidney transplants from HLA-incompatible live donors. N Engl J Med. 2016;374(10):940-50

2. Orandi BJ, Garonzik-Wang JM, Massie AB, Zachary AA, Montgomery JR, Van Arendonk KJ, Stegall MD, Jordan SC, Oberholzer J, Dunn TB, et al. Quantifying the risk of incompatible kidney transplantation: a multicenter study. Am J Transplant Off J Am Soc Transplant Am Soc Transplant Surg. 2014;14(7):1573-80.

3. Bentall A, Cornell LD, Gloor JM, Park WD, Gandhi MJ, Winters JL, Chedid MF, Dean PG, Stegall MD. Five-year outcomes in living donor kidney transplants with a positive crossmatch. Am J Transplant Off J Am Soc Transplant Am Soc Transplant Surg. 2013;13(1):76-85.

4. Haririan A, Nogueira J, Kukuruga D, Schweitzer E, Hess J, Gurk-Turner C, Jacobs S, Drachenberg C, Bartlett S, Cooper M. Positive cross-match living donor kidney transplantation: longer-term outcomes. Am J Transplant Off J Am Soc Transplant Am Soc Transplant Surg. 2009;9(3):536-42.

5. Garonzik Wang JM, Montgomery RA, Kucirka LM, Berger JC, Warren DS, Segev DL. Incompatible live-donor kidney transplantation in the United States: results of a national survey. Clin J Am Soc Nephrol. 2011:6(8):2041-6.

6. Lefaucheur C, Loupy A, Hill GS, Andrade J, Nochy D, Antoine C, Gautreau C, Charron D, Glotz D, Suberbielle-Boissel C. Preexisting donor-specific HLA antibodies predict outcome in kidney transplantation. J Am Soc Nephrol. 2010;21(8):1398-406

7. Loupy A, Lefaucheur C, Vernerey D, Prugger C, Duong van Huyen JP, Mooney N, Suberbielle C, Fremeaux-Bacchi V, Mejean A, Desgrandchamps F, et al. Complement-binding anti-HLA antibodies and kidney-allograft survival. N Engl J Med. 2013;369(13):1215-26.

8. Schinstock CA, Gandhi M, Cheungpasitporn W, Mitema D, Prieto M, Dean P, Cornell L, Cosio F, Stegall M. Kidney transplant with low levels of DSA or low positive B-flow Crossmatch: an underappreciated option for highly sensitized transplant candidates. Transplantation. 2017;101(10):2429-39. 
9. Amrouche L, Aubert O, Suberbielle C, Rabant M, Van Huyen JD, Martinez F, Sberro-Soussan R, Scemla A, Tinel C, Snanoudj R, et al. Long-term outcomes of kidney transplantation in patients with high levels of preformed DSA: the Necker high-risk transplant program. Transplantation. 2017;101(10):2440-8.

10. Cecka JM. Calculated PRA (CPRA): the new measure of sensitization for transplant candidates. Am J Transplant Off J Am Soc Transplant Am Soc Transplant Surg. 2010;10(1):26-9.

11. Reinsmoen NL, Lai CH, Vo A, Cao K, Ong G, Naim M, Wang Q, Jordan SC. Acceptable donor-specific antibody levels allowing for successful deceased and living donor kidney transplantation after desensitization therapy. Transplantation. 2008;86(6):820-5.

12. Mizutani K, Terasaki P, Hamdani E, Esquenazi V, Rosen A, Miller J, Ozawa M. The importance of anti-HLA-specific antibody strength in monitoring kidney transplant patients. Am J Transplant Off J Am Soc Transplant Am Soc Transplant Surg. 2007;7(4):1027-31.

13. Gloor JM, Winters JL, Cornell LD, Fix LA, DeGoey SR, Knauer RM, Cosio FG, Gandhi MJ, Kremers W, Stegall MD. Baseline donor-specific antibody levels and outcomes in positive crossmatch kidney transplantation. Am J Transplant Off J Am Soc Transplant Am Soc Transplant Surg. 2010;10(3):582-9.

14. Okada D, Okumi M, Kakuta Y, Unagami K, lizuka J, Takagi T, Ishida H, Tanabe $K$. Outcome of the risk-stratified desensitization protocol in donorspecific antibody-positive living kidney transplant recipients: a retrospective study. Transpl Int. 2018;31(9):1008-17

15. Buttigieg J, Ali H, Sharma A, Halawa A. Positive Luminex and negative flow cytometry in kidney transplantation: a systematic review and metaanalysis. Nephrol Dial Transplant. 2018;34(11):1950-60.

16. Solez K, Colvin RB, Racusen LC, Haas M, Sis B, Mengel M, Halloran PF, Baldwin W, Banfi G, Collins AB, et al. Banff 07 classification of renal allograft pathology: updates and future directions. Am J Transplant Off J Am Soc Transplant Am Soc Transplant Surg. 2008;8(4):753-60.

17. Levey AS, Stevens LA, Schmid CH, Zhang YL, Castro AF 3rd, Feldman HI, Kusek JW, Eggers P, Van Lente F, Greene T, et al. A new equation to estimate glomerular filtration rate. Ann Intern Med. 2009;150(9):604-12.

18. Kotton CN, Kumar D, Caliendo AM, Asberg A, Chou S, Danziger-Isakov L, Humar A, Transplantation Society International CMVCG. Updated international consensus guidelines on the management of cytomegalovirus in solid-organ transplantation. Transplantation. 2013;96(4):333-60.

19. Pankhurst L, Hudson A, Mumford L, Willicombe M, Galliford J, Shaw O, Thuraisingham R, Puliatti C, Talbot D, Griffin S, et al. The UK National Registry of $\mathrm{ABO}$ and $\mathrm{HLA}$ antibody incompatible renal transplantation: Pretransplant factors associated with outcome in 879 transplants. Transplant Direct. 2017;3(7):e181.

20. Marfo K, Lu A, Ling M, Akalin E. Desensitization protocols and their outcome. Clin J Am Soc Nephrol. 2011;6(4):922-36.

21. Viglietti D, Bouatou Y, Kheav VD, Aubert O, Suberbielle-Boissel C, Glotz D, Legendre C, Taupin JL, Zeevi A, Loupy A, et al. Complement-binding antiHLA antibodies are independent predictors of response to treatment in kidney recipients with antibody-mediated rejection. Kidney Int. 2018;94(4): 773-87.

22. Malheiro J, Tafulo S, Dias L, Martins S, Fonseca I, Beirao I, Castro-Henriques A, Cabrita A. Determining donor-specific antibody C1q-binding ability improves the prediction of antibody-mediated rejection in human leucocyte antigen-incompatible kidney transplantation. Transpl Int. 2017; 30(4):347-59.

23. Juhl D, Marget M, Hallensleben M, Gorg S, Ziemann M. Assignment of C1qbinding HLA antibodies as unacceptable HLA antigens avoids positive CDCcrossmatches prior to transplantation of deceased donor organs. Transpl Immunol. 2017:41:17-21.

24. Waiser J, Budde K, Schutz M, Liefeldt L, Rudolph B, Schonemann C, Neumayer HH, Lachmann N. Comparison between bortezomib and rituximab in the treatment of antibody-mediated renal allograft rejection. Nephrol Dial Transplant. 2012;27(3):1246-51.

25. Walsh RC, Everly JJ, Brailey P, Rike AH, Arend L, Mogilishetty G, Govil A, Roy-Chaudhury P, Alloway RR, Woodle ES. Proteasome inhibitor-based primary therapy for antibody-mediated renal allograft rejection. Transplantation. 2010;89(3):277-84.

26. Everly MJ, Everly JJ, Susskind B, Brailey P, Arend LJ, Alloway RR, RoyChaudhury P, Govil A, Mogilishetty G, Rike AH, et al. Bortezomib provides effective therapy for antibody- and cell-mediated acute rejection. Transplantation. 2008;86(12):1754-61.
27. Moreno Gonzales MA, Gandhi MJ, Schinstock CA, Moore NA, Smith BH, Braaten NY, Stegall MD. 32 doses of Bortezomib for desensitization is not well tolerated and is associated with only modest reductions in anti-HLA antibody. Transplantation. 2017;101(6):1222-7.

28. Woodle ES, Shields AR, Ejaz NS, Sadaka B, Girnita A, Walsh RC, Alloway RR, Brailey P, Cardi MA, Abu Jawdeh BG, et al. Prospective iterative trial of proteasome inhibitor-based desensitization. Am J Transplant Off J Am Soc Transplant Am Soc Transplant Surg. 2015;15(1):101-18.

29. Parajuli S, Muth BL, Turk JA, Astor BC, Mohammed M, Mandelbrot DA, Djamali A. In kidney transplant recipients with a positive virtual Crossmatch, high PRA was associated with lower incidence of viral infections. Transplantation. 2016;100(3):655-61.

30. Riella LV, Safa K, Yagan J, Lee B, Azzi J, Najafian N, Abdi R, Milford E, Mah H, Gabardi S, et al. Long-term outcomes of kidney transplantation across a positive complement-dependent cytotoxicity crossmatch. Transplantation. 2014;97(12):1247-52

31. Orandi BJ, Luo X, King EA, Garonzik-Wang JM, Bae S, Montgomery RA, Stegall MD, Jordan SC, Oberholzer J, Dunn TB, et al. Hospital readmissions following HLA-incompatible live donor kidney transplantation: a multicenter study. Am J Transplant Off J Am Soc Transplant Am Soc Transplant Surg. 2018;18(3):650-8.

\section{Publisher's Note}

Springer Nature remains neutral with regard to jurisdictional claims in published maps and institutional affiliations.

\section{Ready to submit your research? Choose BMC and benefit from:}

- fast, convenient online submission

- thorough peer review by experienced researchers in your field

- rapid publication on acceptance

- support for research data, including large and complex data types

- gold Open Access which fosters wider collaboration and increased citations

- maximum visibility for your research: over $100 \mathrm{M}$ website views per year

At $\mathrm{BMC}$, research is always in progress.

Learn more biomedcentral.com/submissions 